\title{
Some New Intrinsic Topologies on Complete Lattices and the Cartesian Closedness of the Category of Strongly Continuous Lattices
}

\author{
Xiuhua Wu, ${ }^{1}$ Qingguo $\mathrm{Li}^{2}{ }^{2}$ and Dongsheng $\mathrm{Zhao}^{3}$ \\ ${ }^{1}$ Institute of Mathematics and Physics, Central South University of Forestry and Technology, Changsha 410004, China \\ ${ }^{2}$ College of Mathematics and Econometrics, Hunan University, Changsha 410082, China \\ ${ }^{3}$ Mathematics and Mathematics Education, National Institute of Education, Nanyang Technological University, \\ 1 Nanyang Walt, Singapore 637616
}

Correspondence should be addressed to Qingguo Li; liqingguoli@yahoo.com.cn

Received 2 December 2012; Accepted 20 January 2013

Academic Editor: Turgut Öziş

Copyright (C) 2013 Xiuhua Wu et al. This is an open access article distributed under the Creative Commons Attribution License, which permits unrestricted use, distribution, and reproduction in any medium, provided the original work is properly cited.

We prove some new characterizations of strongly continuous lattices using two new intrinsic topologies and a class of convergences. Lastly we show that the category of strongly continuous lattices and Scott continuous mappings is cartesian closed.

\section{Introduction and Preliminaries}

The theory of continuous lattices was first introduced by Scott in 1972 (see [1]) and has been studied extensively by many people from various different fields due to its strong connections to computer science, general topology, algebra, and logics (see [2]). Later the continuous lattices were generalized to various other classes of ordered structures for different motivations, for example, the $L$-domains [3], generalized continuous lattices and hypercontinuous lattices [4], and the most general, continuous dcpos. In [5], in order to get more general results on the relationship between prime and pseudo-prime elements in a complete lattice, two new classes of lattices related to continuous lattices, namely, the semicontinuous lattices and the strongly continuous lattices, were introduced. The semicontinuous lattices were later generalized to $Z$-semicontinuous posets in [6]. Liu and Xie introduced several categories of semicontinuous lattice trying to construct a Cartesian closed category of semicontinuous lattices $($ see $[7,8])$. In $[9,10]$, the first two authors studied other properties of semicontinuous lattices and proved a characterization of semicontinuous lattices in terms of the $S$-open subsets (to be defined later in this paper). Strongly continuous lattices form a proper subclass of continuous lattices. There is some fine connection between strong continuity and distributivity: every distributive continuous lattice is strongly continuous; every Noetherian (in particular, finite) strongly continuous lattice is distributive; a strongly continuous lattice in which the way-below relation is multiplicative is distributive. It has been know for a long time that the category CONT of continuous lattices and Scott continuous mappings is cartesian closed [2, Theorem II-2.12]. It is thus natural to ask if the subcategory of strongly continuous lattices and that of distributive continuous lattices are cartesian closed as well. In this paper we will give a positive answer to these questions. New characterizations of strongly continuous lattices based on some new topologies and convergence of nets are also obtained.

In Section 2, we introduce two new intrinsic topologies on complete lattices, which are then used to formulating a new characterization for strongly continuous lattices. In Section 3, we introduce the lim-inf $f_{S}$ convergence of nets and show that a complete lattice is strongly continuous if and only if the lim-inf $f_{S}$ convergence on the lattice is topological. In Section 4, we define several new types of mappings between complete lattices and study the relationship among $S$-continuous mappings, Scott continuous mappings, strongly continuous mappings, and semicontinuous mappings. In the last section, we consider the category SCONT of strongly continuous lattices and prove that SCONT is Cartesian 
closed. It is also pointed out that the subcategory DCONT of distributive continuous lattices is cartesian closed. For most of the basic definitions and results on continuous lattices we refer to the book [2].

Let $P$ be a poset. For any $A \subseteq P$, define $\uparrow A=\{x \in P$ : $x \geq y$ for some $y \in A\}$. We also write $\uparrow\{x\}$ as $\uparrow x$. The sets $\downarrow A$ and $\downarrow x$ are defined dually.

A subset $A$ of $P$ is called an upper (lower) set if $A=\uparrow$ $A(A=\downarrow A)$. A subset $D$ of $P$ is directed provided it is nonempty, and every finite subset of $D$ has an upper bound in $D$. An ideal of $P$ is a directed lower set.

Let $x, y \in P$. We say that $x$ is way-below $y$, written $x \ll y$, if for any directed subset $D$ with $\bigvee D$ exists and $\bigvee D \geq y$, there is $d \in D$ such that $x \leq d$. Let $\downarrow_{\ll} x=\{a \in P: a \ll x\}$ and let $\uparrow_{\ll} x=\{a \in P: x \ll a\}$. A complete lattice $P$ is called a continuous lattice if for every element $x \in P, x=\bigvee \downarrow_{\ll} x$. It is well known that for any complete lattice $P$ and $x \in P, \downarrow_{\ll} x$ is an ideal.

Definition 1 (see [11]). An ideal $I$ of a lattice $P$ is called semiprime if for all $x, y, z \in P, x \wedge y \in I$ and $x \wedge z \in I$ imply $x \wedge(y \vee z) \in I$. of $P$.

We use $R d(P)$ to denote the family of all semiprime ideals

Definition 2 (see [5]). Let $P$ be a complete lattice. Define the relation $\Leftarrow$ on $P$ as follows: for any $x, y \in P, x \Leftarrow y$ if for any semiprime ideal $I$ of $P, y \leq \bigvee I$ implies $x \in I$. For each $x \in P$, we write

$$
\begin{aligned}
& \Downarrow x=\{y \in P: y \Longleftarrow x\}, \quad \Uparrow x=\{y \in P: x \Longleftarrow y\} . \quad \text { (1) } \\
& \text { For any } A \subseteq P, \text { let } \Downarrow A=\bigcup_{x \in A} \Downarrow x \text { and let } \Uparrow A=\bigcup_{x \in A} \Uparrow \\
& x .
\end{aligned}
$$

Definition 3 (see [5]). A complete lattice $P$ is called semicontinuous, if for any $x \in P$,

$$
x \leq \bigvee \Downarrow x .
$$

$P$ is called strongly continuous, if $x=\bigvee \Downarrow x$ for any $x \in P$.

Theorem 4 (see [5]). If $P$ is a semicontinuous lattice, then the relation $\Leftarrow$ has the interpolation property; that is, $x \Leftarrow y$ implies the existence of $z \in P$ such that $x \Leftarrow z \Leftarrow y$.

Lemma 5 (see [5]). Let $P$ be a complete lattice; then $\Downarrow x$ is a semiprime ideal for each $x \in P$.

\section{Strong Continuity via Topology}

For any complete lattice $P, U \subseteq P$ is called Scott open if and only if $U=\uparrow U$ and for any directed set $D \subseteq P, \bigvee D \in$ $U$ implies $U \cap D \neq \emptyset$. All Scott open subsets of $P$ form a topology, called the Scott topology, denoted by $\sigma(P)$ [2]. One of the classic characterizations of continuous lattices is that a complete lattice $P$ is continuous if and only if $(\sigma(P), \subseteq)$ is completely distributive. This result was later generalized to continuous posets [2]. In the current section we introduce two new intrinsic topologies on complete lattices and use them to establish some new characterizations for strongly continuous lattices.

Definition 6. A subset $U$ of a complete lattice $P$ is called $S$ open if and only if $U=\uparrow U$ and for any semiprime ideal $I$ of $P, \bigvee I \in U$ implies $U \cap I \neq \emptyset$.

We use $\kappa(P)$ to denote the family of all $S$-open subsets of $P$; it is easy to verify that $\kappa(P)$ forms a topology on $P$, called the $S$-topology.

Definition 7. A subset $U$ of a complete lattice is called $T$-open if and only if $U=\Uparrow U$.

We use $\tau(P)$ to denote the family of all $T$-open subsets of the complete lattice $P$.

Remark 8. As $x \Leftarrow y \leq z$ implies $x \Leftarrow z$, it follows that every $T$-open set is an upper set. Furthermore, for any complete lattice $P, \tau(P)$ forms a topology on $P$. Obviously $\tau(P)$ contains the empty set and $P$ and is closed under arbitrary union. Let $U, V \in \tau(P)$. We show that $U \cap V \in \tau(P)$. For any $x \in U \cap V$, $x \in U, x \in V$, thus there exist $m \in U, n \in V$ such that $m \Leftarrow x$ and $n \Leftarrow x$. Therefore $m \vee n \Leftarrow x$. Note that $m \vee n \in U \cap V$, thus $x \in \Uparrow(U \cap V)$. Conversely, if $x \in \Uparrow(U \cap V)$, then there exists $y \in U \cap V$ such that $y \Leftarrow x$. Since $U=\Uparrow U$ and $V=\Uparrow V$, so $x \in U$ and $x \cap V$, thus $x \in U \cap V$. Thus $U \cap V=\Uparrow(U \cap V)$, which belongs to $\tau(P)$. Hence $\tau(P)$ forms a topology on $P$.

We call the topology $\tau(P)$ the T-topology. Obviously every Scott open set is $S$-open. The reverse conclusion does not need to be true. There is not any inclusion relation between the $T$-topology and the Scott topology applying to all complete lattices.

Example 9. (1) Let $P=[0,1] \cup\{a, b\}$, where $[0,1]$ is the unit interval; $a$ and $b$ are two distinct elements not in $[0,1]$. The partial order $\leq$ on $P$ is defined by: $0<a<1,0<b<1$; for $x, y \in[0,1], x \leq y$ if $x$ is less than or equal to $y$ according to the usual order on real numbers.

Obviously $P$ is the unique semiprime ideal of $P$, so $\uparrow a$ (in fact, every upper set) is $S$-open. For each $n \in N$, let $c_{n}=$ $1-(1 / n)$. Then $D=\left\{c_{n}\right\}_{n \in N}$ is a directed set such that $\bigvee D=$ $1>a$, but for each $n, c_{n} \notin \uparrow a$. Thus $\uparrow a$ is not Scott open. Also, $\Uparrow(\uparrow a)=P, \uparrow a$ is not $T$-open either.

(2) Let $P=\{0, a, b, c, 1\}$ be the five-element modular lattice. Then clearly $\{a, 1\}$ is Scott open. Again, in this example, $P$ is the only semiprime ideal, so $\Uparrow A=P$ for any nonempty set $A$. Hence $\{a, 1\}$ is not $T$-open.

Lemma 10. (1) If $P$ is a semicontinuous lattice, then $\tau(P) \subseteq$ $\sigma(P) \subseteq \kappa(P)$.

(2) If $P$ is strongly continuous, then $\tau(P)=\sigma(P)=\kappa(P)$.

Proof. (1) It only needs to show that $\tau(P) \subseteq \sigma(P)$. Let $U \in$ $\tau(P)$. For any directed subset $D \subseteq P$ with $\bigvee D \in U$, there exists $a \in U$ such that $a \Leftarrow \bigvee D$. Since $P$ is semicontinuous, $a \Leftarrow \bigvee D \leq \bigvee\{\bigvee \Downarrow d: d \in D\}=\bigvee\left(\bigcup_{d \in D} \Downarrow d\right)$. Note that the union $\bigcup_{d \in D} \Downarrow d$ is still a semiprime ideal, so there is $d \in D$ 
such that $a \in \Downarrow d$. Thus $d \in \Uparrow a \subseteq U$. Therefore $D \cap U \neq \emptyset$, hence $U \in \sigma(P)$. This proves $\tau(P) \subseteq \sigma(P)$.

(2) Now assume that $P$ is strongly continuous. Then $P$ is continuous. In a strongly continuous lattice, the relation $\ll$ and $\Leftarrow$ are the same by the Theorem 2.5 of [5]. Also by [2] in a continuous lattice, every Scott open set $A$ satisfies the condition $A=\uparrow_{\ll} A$, it follows that every Scott open set of $P$ is $T$-open. Now let $U$ be $S$-open. For any directed set $D$ with $\bigvee D \in U$, let $I=\bigcup_{d \in D} \Downarrow d$. Then $I$ is a semiprime ideal such that $\bigvee I=\bigvee D$. Thus, as $U$ is $S$-open, $I \cap U \neq \emptyset$. Hence $D \cap U \neq \emptyset$. Therefore $U$ is Scott open. By (1) we have $\tau(P)=\sigma(P)=\kappa(P)$.

Lemma 11. For any complete lattice $P$, if $\tau(P)=\kappa(P)$ then $P$ is semicontinuous.

Proof. Suppose that $P$ is not semicontinuous. Then there exists $x \in P$ such that $x \not \bigvee \Downarrow x$. Then $x \in U=P \backslash \downarrow(\bigvee \Downarrow$ $x$ ) which is obviously $S$-open. Since $\tau(P)=\kappa(P), U$ is $T$-open. Thus there exists $u \nless \backslash \bigvee \Downarrow x$ such that $u \Leftarrow x$ by Definition 7 , a contradiction. Thus $P$ must be semicontinuous.

Let $P$ be a complete lattice. The long way-below relation $\triangleleft$ on $P$ is defined as follows: for any $x, y \in P, x \triangleleft y$ if and only if for any nonempty subset $B \subseteq P, y \leq \bigvee B$ implies that $x \leq z$ for some $z \in B$. For each $x \in P$, we write

$$
\beta(x)=\{y \in P: y \triangleleft x\} .
$$

Clearly $x \triangleleft y$ implies $x \ll y$. In [12], Raney proved that a complete lattice $P$ is a completely distributive lattice if and only if $x=\bigvee \beta(x)$ for all $x \in P$.

It is well known that a complete lattice $P$ is a continuous if and only if the topology $\sigma(P)$ is a completely distributive lattice [2]. If $P$ is semicontinuous, $\kappa(P)$ is generally not a completely distributive lattice. In Example $9(1), P$ is semicontinuous and $\kappa(P)$ consists of all upper subsets of $P$. Then $(\kappa(P), \supseteq)$ is a complete lattice and the empty set is the largest element of $(\kappa(P), \supseteq)$, but $\emptyset \neq \bigvee \beta(\emptyset)$. Thus $\kappa(P)$ is not a completely distributive lattice.

Proposition 12. Let $(P, \leq)$ be a complete lattice in which the relation $\Leftarrow$ satisfies the interpolation property. Then $\tau(P)$ is a completely distributive lattice.

Proof. By Raney's characterization of completely distributive lattices, we need to show that $E \leq \bigvee \beta(E)$ hold for all $E \in \tau(P)$.

For any $x \in E \in \tau(P)$, by the definition of $\tau(P)$, there exists $y \in E$ such that $x \in \Uparrow y \subseteq E$. Since the relation $\Leftarrow$ satisfies the interpolation property, $\Uparrow y \in \tau(P)$. Now we claim that $\Uparrow y \triangleleft E$. Let $\mathscr{D} \subseteq \tau(P)$ such that $E \leq \bigvee \mathscr{D}$. Since $y \in$ $E$ and $\bigvee \mathscr{D}=\bigcup \mathscr{D}$, there exists $U_{0} \in \mathscr{D}$ such that $y \in U_{0}$. Therefore $\Uparrow y \leq U_{0}$. Hence $\Uparrow y \triangleleft E$. It now follows that $E \leq$ $\bigvee\{\Uparrow y: y \in E\} \leq \bigvee \beta(E)$. This completes the proof.

Lemma 13. If $P$ is a complete lattice such that $\tau(P)=\kappa(P)$, then $P$ is a continuous lattice.

Proof. If $\tau(P)=\kappa(P)$, then $P$ is semicontinuous and $\tau(P)$ is a completely distributive lattice by Lemma 11 and
Proposition 12. By Lemma 10, $\tau(P)=\sigma(P)=\kappa(P)$, thus $(\sigma(P), \subseteq)$ is a completely distributive lattice. It then follows from Theorem II-1.13 of [2] that $P$ is continuous. This completes the proof.

Lemma 14. Let $P$ be a complete lattice. If $\tau(P)=\kappa(P)$, then $P$ is strongly continuous.

Proof. By Lemma 11, $P$ is semicontinuous. Assume that $P$ is not strongly continuous. Then there exists $b \in P$ such that $b<\bigvee \Downarrow b$. Thus $\bigvee \Downarrow b \in P \backslash \downarrow b$ which is obviously $S$ open. By Lemma 5 and Definition 6, there exists $a \in P$ such that $a \Leftarrow b$ and $a \in P \backslash \downarrow b$. By Lemma 13, $a=\bigvee \downarrow_{\ll} a \in$ $P \backslash \downarrow b \in \sigma(P)$. Thus there exists $x \ll a$ such that $x \not b$. By Lemma 10, $P$ is continuous and so $\uparrow_{\ll} x \in \sigma(P)$ for all $x \in P$. Again by Lemma 10 and $\tau(P)=\kappa(P), \tau(P)=\sigma(P)=\kappa(P)$, so $\Uparrow\left(\uparrow_{\ll} x\right)=\uparrow_{\ll} x$. Thus $b \in \Uparrow a \subseteq \Uparrow\left(\uparrow_{\ll} x\right)=\uparrow_{\ll} x$. And then $x \ll b$. So $x \leq b$, which contradicts the assumption on $x$. This completes the proof.

From Lemmas 10 and 14, we obtain the following new characterization of strongly continuous lattices.

Theorem 15. A complete lattice $P$ is strongly continuous if and only if $\tau(P)=\kappa(P)$.

\section{3. lim-inf ${ }_{S}$ Convergence and Strongly Continuous Lattices}

In [2], the lim-inf convergence is introduced, and it is proved that a complete lattice is continuous if and only if the lim-inf convergence on the lattice is topological. This result was later generalized to continuous dcpos and continuous posets (see $[2,13])$. Now we introduce a similar convergence, lim-inf $f_{S}$ convergence, and show that a complete lattice is strongly continuous if and only if the lim-inf $f_{S}$ convergence on the lattice is topological.

Definition 16. A net $\left(x_{j}\right)_{j \in J}$ in a complete lattice $P$ is said to lim-inf f $_{S}$ converge to an element $y \in P$ if there exists a semiprime ideal $I$ of $P$ such that

(1) $\bigvee I \geq y$, and

(2) for any $m \in I, x_{j} \geq m$ holds eventually (i.e., there exists $k \in J$ such that $x_{j} \geq m$ for all $j \geq k$ ).

In this case we write $y \equiv \lim -\inf _{S} x_{j}$.

For any complete lattice $P$, define

$$
\begin{aligned}
& \mathscr{P}=\left\{\left(\left(x_{j}\right)_{j \in J}, x\right):\left(x_{j}\right)_{j \in J}\right. \text { is a net } \\
& \text { with } \left.x \equiv \lim -\inf _{S} x_{j}\right\} \text {. }
\end{aligned}
$$

The class $\mathscr{P}$ is called topological if there is a topology $T$ on $P$ such that $\left(\left(x_{j}\right)_{j \in J}, x\right) \in \mathscr{P}$ if and only if the net $\left(x_{j}\right)_{j \in J}$ converges to $x$ with respect to the topology $T$. 
As in usual cases, associated with $\mathscr{P}$ is a family of sets, which is a topology on $P$ :

$$
\begin{gathered}
\mathscr{O}(\mathscr{P})=\left\{U \subseteq P: U=\uparrow U \text { and whenever }\left(\left(x_{j}\right)_{j \in J}, x\right) \in \mathscr{P}\right. \\
\text { and } \left.x \in U, \text { so eventually } x_{j} \in U\right\} .
\end{gathered}
$$

Proposition 17. For any complete lattice $P, \mathscr{O}(\mathscr{P})=\kappa(P)$.

Proof. First, suppose $U \in \mathscr{O}(\mathscr{P})$. Let $I$ be a semiprime ideal in $P$ with $\bigvee I \in U$. Consider the net $\left(x_{d}\right)_{d \in I}$ with $x_{d}=d$. Now for any $a \in I, x_{d} \geq a$ holds eventually. Thus $\left(\left(x_{d}\right)_{d \in I}, \bigvee I\right) \in \mathscr{P}$. From the definition of $\mathscr{O}(\mathscr{P})$ we conclude that the net $\left(x_{d}\right)_{d \in I}$ must be eventually in $U$, and then there exists $d \in I$ such that $x_{w} \in U$ for all $w \geq d$, whence $D \cap U \neq \emptyset$.

Conversely, suppose $U \in \kappa(P)$. For any $\left(\left(x_{j}\right)_{j \in J}, x\right) \in \mathscr{P}$ such that $x \in U$, by the definition of $\mathscr{P}$, we have $x \leq \bigvee I$ for some semiprime ideal $I$ and for each $u \in I, x_{j} \geq u$ holds eventually. Now $\bigvee I \in U$, so by the definition of $\kappa(P)$, there exists $d \in U$ such that $d \in I$. Then there exists $k \in I$ such that $d \leq x_{j}$ for all $j \geq k$. Thus $x_{j} \in U$ for all $j \geq k$. Hence $x_{j} \in U$ holds eventually. Thus $U \in \mathscr{O}(\mathscr{P})$.

Lemma 18. If $P$ is a complete lattice and $y \in$ int $_{\kappa} \uparrow x$, then $x \Leftarrow y$, where int $_{\kappa} \uparrow x$ denotes the interior of $\uparrow x$ with respect to the S-topology.

Proof. Let $P$ be a complete lattice and $y \in$ int $_{\kappa} \uparrow x$. For any semiprime ideal $I$ with $y \leq \bigvee I$, we have $\bigvee I \in$ int $_{\kappa} \uparrow x$. Thus there exists $d \in\left(\right.$ int $\left._{\kappa} \uparrow x\right) \cap I$. Therefore $x \leq d$ and then $x \in I$. Thus $x \Leftarrow y$.

Proposition 19. Let $P$ be a strongly continuous lattice. Then $x \equiv \lim -\inf _{S} x_{j}$ if and only if the net $\left(x_{j}\right)_{j \in J}$ converges to the element $x$ with respect to $\kappa(P)$. In particular, the lim-inf ${ }_{S}$ convergence is topological.

Proof. By Proposition 17, $\mathscr{O}(\mathscr{P})=\kappa(P)$, so if $x \equiv \lim -\inf _{S} x_{j}$ then $\left(x_{j}\right)_{j \in J}$ converges to the element $x$ with respect to $\kappa(P)$. Conversely, suppose that we have a net $\left(x_{j}\right)_{j \in J}$ which converges to the element $x$ with respect to $\kappa(P)$. For each $y \in \Downarrow x$, we have $x \in \Uparrow y \in \kappa(P)$ from the definition of $\kappa(P)$. Thus there exists $k \in I$ such that $x_{j} \in \Uparrow y$ for all $j \geq k$, and then $y \leq x_{j}$ for all $j \geq k$. Since $P$ is strongly continuous, $x=\bigvee \Downarrow x$. By Lemma $5, \Downarrow x$ is a semiprime ideal. Therefore we have $\left(\left(x_{j}\right)_{j \in J}, x\right) \in \mathscr{P}$. That is, $x \equiv \lim -\inf _{S} x_{j}$.

Lemma 20. Let $P$ be a complete lattice. If the lim-inf $S_{S}$ convergence is topological, then $P$ is strongly continuous.

Proof. By Proposition 17, the topology arising from lim-inf $f_{S}$ convergence is the $S$-topology. Thus if the lim-inf conver- $_{S}$ gence is topological, we must have that $x \equiv \lim -\inf _{S} x_{j}$ if and only if the net $\left(x_{j}\right)_{j \in J}$ converges to the element $x$ with respect to $\kappa(P)$. For any $x \in P$, let $J=\{(U, n, a) \in N(x) \times \mathbb{N} \times P: a \in$ $U$, where $N(x)$ consists of all $S$-open sets containing $x$, and define an order on $J$ to be the lexicographic order on the first two coordinates. That is, $(U, m, a) \leq(V, n, b)$ if and only if
$V$ is a proper subset of $U$ or $U=V$ and $m \leq n$. Let $x_{j}=a$ for each $j=(U, n, a) \in J$. Then it is easy to verify that the net $\left(x_{j}\right)_{j \in J}$ converges to the element $x$ with respect to $\kappa(P)$. Thus $x \equiv \lim -\inf _{S} x_{j}$, and we conclude that there exists a semiprime ideal $I$ such that $x \leq \bigvee I$ and $x_{j} \geq u$ holds eventually for each $u \in I$. Let $d \in I$, then there exists $k=(U, m, a) \in I$ such $(V, n, b)=j \geq k$ implies $d \leq x_{j}=b$. In particular, we have $(U, m+1, b) \geq(U, m, a)=k$ for all $b \in U$. Thus $x \in U \subseteq \uparrow d$. It follows that $I \subseteq \downarrow x$. Furthermore, $x \in \operatorname{int}_{\kappa} \uparrow d$. By Lemma 18, $d \Leftarrow x$ and then $I \subseteq \Downarrow x$. Therefore $x=\bigvee I \leq \bigvee \Downarrow x$. Since $I$ is a semiprime ideal with a supremum greater than or equal to $x$, it follows that $\Downarrow x \subseteq I$. Hence $I=\Downarrow x$ and so $x=\bigvee \Downarrow x$. All these show that $P$ is strongly continuous.

What we now have proved is the following characterization of strongly continuous lattices.

Theorem 21. Let $P$ be a complete lattice, then the following statements are equivalent.

(1) $P$ is strongly continuous.

(2) The lim-inf ${ }_{S}$ convergence is the convergence for the $S$ topology; that is, for all $x \in P$ and all nets $\left(x_{j}\right)_{j \in J}$ in $P$ $x \equiv \lim -\inf _{S} x_{j}$ if and only if the net $\left(x_{j}\right)_{j \in J}$ converges to the element $x$ with respect to $\kappa(P)$.

(3) The lim-inf ${ }_{S}$ convergence is topological.

\section{Continuous Mappings}

In this section, we will investigate the relations among semicontinuous mappings, strongly semicontinuous mappings, Scott continuous mappings, and $S$-continuous mappings. Firstly recall from [2] that a mapping $f: L \rightarrow M$ is said to be Scott continuous, if $f$ is continuous from topological spaces $(L, \sigma(L))$ to $(M, \sigma(M))$. It is known that $f$ is Scott continuous if and only if $f$ preserves all directed suprema (see [2]). Now we give some new basic definitions.

Definition 22. Let $L, M$ be complete lattices. An order preserving mapping $f: L \rightarrow M$ is called a semicontinuous mapping, if $f$ preserves suprema of semiprime ideals; $f$ is called a strongly semicontinuous mapping, if $f$ is semicontinuous and for any $I \in R d(L), \downarrow f(I) \in R d(M)$. A mapping $f: L \rightarrow M$ is called $S$-continuous if it is continuous from topological spaces $(L, \kappa(L))$ to $(M, \kappa(M))$.

Lemma 23. Let $L, M$ be complete lattices. If $f: L \rightarrow M$ is $S$-continuous, then $f$ is order-preserving.

Proof. Let $x \leq y$ in L. Suppose that $f(x) \nless f(y)$; then the $S$ open set $V=M \backslash \downarrow f(y)$ contains $f(x)$. Thus $U=f^{-1}(V)$ is a $S$-open neighborhood of $x$ not containing $y$. But then $x \not$ $y$ as $U$ is an upper set, a contradiction. Thus $x \leq y$ implies $f(x) \leq f(y)$.

Lemma 24. Let $P$ be a complete lattice and $A=\downarrow A \subseteq P$. Then $A$ is S-closed if and only if $\bigvee I \in A$ holds for any $I \in R d(P)$ with $I \subseteq A$. 
Lemma 25. Let $L, M$ be complete lattices. If $f: L \rightarrow M$ is a strongly semicontinuous mapping, then $f$ is S-continuous.

Proof. Let $A$ be an $S$-closed subset of $M$. First, $f^{-1}(A)$ is a lower set because $f$ is order preserving and $A$ is a lower set. For arbitrary semiprime ideal $I \subseteq f^{-1}(A)$, we have $\downarrow f(I) \subseteq$ A. Since $f$ is strongly semicontinuous, $f(\bigvee I)=\bigvee f(I)=\bigvee \downarrow$ $f(I)$ and $\downarrow f(I) \in R d(M)$. By Lemma 24, $\bigvee f(I)=\bigvee \downarrow f(I) \epsilon$ $A$. Therefore $\bigvee I \in f^{-1}(A)$. Again by Lemma $24, f^{-1}(A)$ is $S$ closed. Thus $f$ is $S$-continuous. This proves our result.

Proposition 26. Let $f: L \rightarrow M$ be an order preserving mapping from a strongly continuous lattice $L$ to a complete lattice $M$. Then $f$ is Scott continuous if and only if $f$ is semicontinuous.

\section{Proof. Necessity. Obvious.}

Sufficiency. Let $D$ be arbitrary directed subset of $L$. Since $f$ is order-preserving, $\bigvee f(D) \leq f(\bigvee D)$. Suppose that $f(\bigvee D) \not \bigvee f(D)$. Then $f(\bigvee D) \in U=M \backslash \downarrow(\bigvee f(D)) \in$ $\sigma(M)$. Thus $\bigvee D=\bigvee \downarrow D \in f^{-1}(U)$. Since $L$ is a strongly continuous lattice, by Theorem 2.5 in [5], there exists a semiprime ideal $I \subseteq \downarrow D$ such that $\bigvee I=\bigvee \downarrow D \in f^{-1}(U)$ Hence $f(\bigvee I)=\bigvee f(I) \in U \in \sigma(M)$. It then follows that $f(I) \cap U \neq \emptyset$. Thus there exist $t \in U$ and $d \in D$ such that $t \leq f(d)$. Therefore $f(d) \in U$. That is, $f(d) \nless \bigvee f(D)$, which contradicts $\bigvee f(D) \leq f(\bigvee D)$. Hence $\bigvee f(D)=f(\bigvee D)$ holds for all directed set $D$; thus $f$ is Scott continuous.

Proposition 27. Let $f: L \rightarrow M$ be a map from a strongly continuous lattice $L$ to a distributive lattice $M$. Then the following conditions are equivalent:

(1) $f$ is Scott continuous;

(2) $f$ is strongly semicontinuous;

(3) $f$ is S-continuous;

(4) $f$ is semicontinuous.

Proof. $(1) \Rightarrow(2)$. Let $f$ be Scott continuous. Then $f$ is order preserving and preserves the supremum of all semiprime ideals. Let $I \in R d(L)$. Then $\downarrow f(I)$ is an ideal of $M$. Since $M$ is distributive, $\downarrow f(I)$ is the semiprime ideal of $M$.

(2) $\Rightarrow$ (3) follows from Lemma 25 .

(3) $\Rightarrow(4)$. Let $f: L \rightarrow M$ be $S$-continuous. By Lemma 23, $f$ is order-preserving. Let $I$ be any semiprime ideal of $L$. Thus $\bigvee f(I) \leq f(\bigvee I)$. Assume that $\bigvee f(I)<f(\bigvee I)$. Then $f(\bigvee I) \in U=M \backslash \downarrow(\bigvee f(I))$ which is obviously $S$-open. Hence $\bigvee I \in f^{-1}(U) \in \kappa(L)$. Therefore $I \cap f^{-1}(U) \neq \emptyset$ and then there exists $d \in I$ such that $f(d) \in U$. That is, $f(d) \not \bigvee f(I)$ a contradiction.

$(1) \Leftrightarrow(4)$ follows from Proposition 26 .

Corollary 28. Let $L, M$ be strongly continuous lattice. Then $f$ is Scott continuous if and only if $f$ is semicontinuous.

The following proposition follows directly from Lemma 23 and Theorem 1 of [7].
Proposition 29. Let $L$ be a semicontinuous lattice and let $M$ be a complete lattice. If there is a surjective S-continuous mapping $f: L \rightarrow M$ that preserves the relation $\Leftarrow$, then $M$ is semicontinuous.

\section{The Cartesian Closedness of the Category of Strongly Continuous Lattices}

Let SCONT denote the category of all strongly continuous lattices and semicontinuous mappings between them. By Corollary 28, the morphisms of SCONT are the Scott continuous mappings. Thus SCONT is a full subcategory of the category CONT of continuous lattices and Scott continuous mappings. Given two complete lattices $D$ and $E$ we will use $0_{E}$ to denote the least element of $E,[D \rightarrow E]$ to denote the set of all order-preserving maps from $D$ to $E,[D, E]$ to denote the set of all Scott continuous mappings from $D$ to $E$, and $[D \hookrightarrow E]$ to denote the set of all semicontinuous mappings from $D$ to $E$. Obviously, $[D, E] \subseteq[D \hookrightarrow E] \subseteq[D \rightarrow E]$, and they are all posets with respect to the pointwise order. In particular, $[D, E]$ is a complete lattice. It is well known that the category CONT is Cartesian closed [2, Theorem II-2.12]. Thus it is natural to ask whether SCONT is cartesian closed as well. In this section we will give a positive answer to this question.

Lemma 30. For any two complete lattices $D$ and $E$, the set $[D \hookrightarrow E]$ is closed under taking supremum in $[D \rightarrow E]$; thus it is a complete lattice.

Proof. Let $\perp(x)=0_{E}$ for all $x \in D$. It is easy to show that $\perp$ is the bottom element of $[D \hookrightarrow E]$.

Let $\mathscr{F} \subseteq[D \hookrightarrow E]$ and $h(x)=\bigvee_{f \in \mathscr{F}} f(x)$ for any $x \in D$. Then $h: D \rightarrow E$ is the supremum of $\mathscr{F}$ in $[D, E]$. Now for semiprime ideal $I$ in $D$, we have

$$
\begin{aligned}
\bigvee h(I) & =\bigvee_{x \in I} h(x)=\bigvee_{x \in I} \bigvee_{f \in \mathscr{F}} f(x)=\bigvee_{f \in \mathscr{F}} \bigvee f(I) \\
& =\bigvee_{f \in \mathscr{F}} f(\bigvee I)=h(\bigvee I) .
\end{aligned}
$$

Thus $\bigvee \mathscr{F}=h \in[D \hookrightarrow E]$.

Let $D$ and $E$ be two complete lattices. For every $x \in D$ and $y \in E$, define the interpolating step function $[x \hookrightarrow y]$ : $D \rightarrow E$ by

$$
[x \hookrightarrow y](a)= \begin{cases}y, & x \ll a, \\ 0_{E}, & \text { otherwise }\end{cases}
$$

Lemma 31. Let $D$ be a complete lattice for which the waybelow relation $\ll$ satisfies the interpolation property. Then for all $x \in D$ and $y \in E,[x \hookrightarrow y]$ is Scott continuous.

Proof. Clearly $[x \hookrightarrow y]$ is order preserving. Let $I$ be any ideal of $D$. If $x \ll \bigvee I$, then $[x \hookrightarrow](\bigvee I)=y$. Since the way below relation $\ll$ satisfies the interpolation property, there exists $c \in D$ such that $x \ll c \ll \bigvee I$. Thus $c \in I$. 


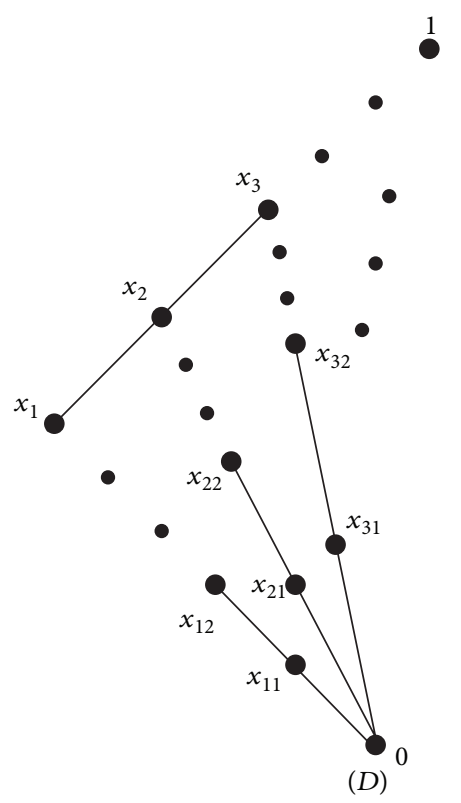

FIgURe 1

Therefore $\bigvee[x \hookrightarrow y](I)=y=[x \hookrightarrow y](\bigvee I)$. If $x \ll \bigvee I$, then $x \ll z$ for all $z \in I$. Hence $\bigvee[x \hookrightarrow y](I)=[x \hookrightarrow y](\bigvee I)=0_{E}$. Therefore $[x \hookrightarrow y]$ preserves supremum of arbitrary ideal. So it is Scott continuous.

The following example illustrates that the assumption that the way below relation satisfies the interpolation property in Lemma 31 is necessary.

Example 32. Let $D=\{0,1\} \cup\left\{x_{i}: i=1, \ldots, n, \ldots\right\} \cup\left\{x_{i j}\right.$ : $i, j=1, \ldots, n, \ldots\}$ and $E=\left\{0_{E}, 1_{E}\right\}$ with $0_{E}<1_{E}$. The order $\leq$ on $D$ is given by (see also Figure 1)

(i) $0 \leq x \leq 1$ for all $x \in D$;

(ii) for each $n, x_{n} \leq x_{n+1}$;

(iii) for each $x_{i}$ and each $n, x_{i, n} \leq x_{i, n+1} \leq x_{i}$.

Then $x_{1} \ll 1$ in $D$, but $x_{1} \ll x_{i}$ for $i=2, \ldots, n, \ldots$. Thus the way-below relation $\ll$ on $D$ does not satisfy the interpolation property. Consider the mapping $\left[\begin{array}{lll}x_{1} & \hookrightarrow & 1_{E}\end{array}\right]$. Let $I=D \backslash\{1\}$. Then $\bigvee I=1 .\left[x_{1} \hookrightarrow 1_{E}\right](\bigvee I)=1_{E}$, but $\bigvee\left[x_{1} \hookrightarrow 1_{E}\right](I)=0_{E} \neq\left[x_{1} \hookrightarrow 1_{E}\right](\bigvee I)$. Thus $\left[x_{1} \hookrightarrow 1_{E}\right]$ is not Scott continuous.

Lemma 33. Let $D$ be a complete lattice for which the waybelow relation «satisfies the interpolation property and $f \in$ $[D, E]$. Then for all $x \in D$ and $y \in E, y \ll f(x)$ implies $[x \hookrightarrow y] \ll f$.

Proof. Let $\mathscr{I}$ be any ideal of $[D, E]$ with $f \leq \bigvee \mathscr{I}$. Then $f(z) \leq \bigvee_{g \in \mathcal{F}} g(z)$ for all $z \in D$. Then $y \ll f(x) \leq \bigvee_{g \in \mathcal{F}} g(x)$. Since $\{g(x): g \in \mathscr{I}\}$ is directed, there exists $g_{*} \in \mathscr{I}$ such that $y \leq g_{*}(x)$. Now we claim that $[x \hookrightarrow y] \leq g_{*}$.

For arbitrary $a \in D, x \ll a$ implies $x \leq a$ and $[x \hookrightarrow$ $y](a)=y \leq g_{*}(x) \leq g_{*}(a)$. If $x \ll a$, then $[x \hookrightarrow y](a)=0_{E} \leq$ $g_{*}(a)$. Therefore $[x \hookrightarrow y](a) \leq g_{*}(a)$ for all $a \in D$. That is, $[x \hookrightarrow y] \leq g_{*}$. This proves our claim. Hence $[x \hookrightarrow y] \in \mathscr{I}$. Therefore $[x \hookrightarrow y] \ll f$.

The following proposition can be found in [2].

Proposition 34 (see [2]). Let $D$ and $E$ be continuous lattices. Then for each $f \in[D, E], f=\bigvee\{[x \hookrightarrow y]: y \ll f(x)\}$; hence $[D, E]$ is a continuous lattice.

The preceding proposition yields a characterization of the way-below relation on function spaces via interpolating step functions.

Corollary 35. Let $D$ and $E$ be continuous lattices and $f, g \in$ $[D, E]$. Then $g \ll f$ holds in $[D, E]$ if and only if there exist $x_{i} \in D, y_{i} \in E$, for $i=1,2, \ldots, n$, such that

$$
y_{i} \ll f\left(x_{i}\right), \quad g \leq \bigvee_{i=1}^{n}\left[x_{i} \hookrightarrow y_{i}\right] .
$$

Corollary 36. If $D$ and $E$ are continuous lattices and $g \ll f$ holds in $[D, E]$, then $g(a) \ll f(a)$ holds for all $a \in D$.

Proof. With the notation of the previous corollary, we have that

$$
g \leq \bigvee_{i=1}^{n}\left\{\left[x_{i} \hookrightarrow y_{i}\right]: y_{i} \ll f\left(x_{i}\right)\right\} .
$$

Let $a \in D$. For any $i$, if $x_{i} \ll a$, then $\left[x_{i} \hookrightarrow y_{i}\right](a)=y_{i} \ll$ $f\left(x_{i}\right) \leq f(a)$. Hence $\left[x_{i} \hookrightarrow y_{i}\right](a) \ll f(a)$. If $x_{i} \ll a$, then $\left[x_{i} \hookrightarrow y_{i}\right](a)=0_{E} \ll f(a)$. Thus $g(a) \leq \bigvee_{i=1}^{n}\left\{\left[x_{i} \hookrightarrow y_{i}\right](a)\right.$ : $\left.y_{i} \ll f\left(x_{i}\right)\right\} \ll f(a)$. Therefore $g(a) \ll f(a)$. This completes our proof.

Lemma 37. Let $D$ be continuous and let $E$ be strongly continuous. Then for any $f \in[D, E], \downarrow_{\ll} f=\{g \in[D, E]$ : $g \ll f\}$ is a semiprime ideal of $[D, E]$.

Proof. Obvious $\downarrow_{\ll} f$ is an ideal because $[D, E]$ is a complete lattice. Let $h \wedge g_{1}, h \wedge g_{2} \in \downarrow_{\ll} f$. By Proposition 34, $[D, E]$ is continuous, so the relation $\ll$ satisfies the interpolation property. Thus there exists $f^{*} \in \downarrow_{\ll} f$ such that $h \wedge g_{1} \ll$ $f^{*} \ll f$ and $h \wedge g_{2} \ll f^{*} \ll f$. For arbitrary $a \in D$, by Corollary 36, $\left(h \wedge g_{1}\right)(a)=h(a) \wedge g_{1}(a) \ll f^{*}(a)$, $\left(h \wedge g_{2}\right)(a)=h(a) \wedge g_{2}(a) \ll f^{*}(a)$. Since $E$ is strongly continuous, $h(a) \wedge g_{1}(a) \Leftarrow f^{*}(a)$ and $h(a) \wedge g_{2}(a) \Leftarrow f^{*}(a)$. Thus $h(a) \wedge\left(g_{1}(a) \vee g_{2}(a)\right) \Leftarrow f^{*}(a)$. And then $\left(h \wedge\left(g_{1} \vee\right.\right.$ $\left.\left.g_{2}\right)\right)(a)=h(a) \wedge\left(g_{1}(a) \vee g_{2}(a)\right) \ll f^{*}(a)$. Since $a$ is arbitrary, $h \wedge\left(g_{1} \vee g_{2}\right) \leq f^{*} \ll f$. And follows $h \wedge\left(g_{1} \vee g_{2}\right) \ll f$. Thus $\downarrow_{\ll} f$ is a semiprime ideal of $[D, E]$. This completes our proof.

Theorem 38. If $D$ is a continuous lattice and $E$ is a strongly continuous lattice, then $[D, E]$ is strongly continuous.

Proof. By Proposition 34, $[D, E]$ is a continuous lattice and $f=\bigvee \downarrow_{\ll} f$ for all $f \in[D, E]$, so $[D, E]$ is a semicontinuous lattice. Let $f, g \in[D, E]$ with $g \Leftarrow f=\bigvee \downarrow_{\ll} f$. By Lemma 37, $\downarrow_{\ll} f$ is an semiprime ideal, so $g \in \downarrow_{\ll} f$, that is, $g \ll f$. By 
Theorem 2.5 of $[5],[D, E]$ is a strongly continuous lattice. This completes our proof.

For cartesian closedness we adopt the elementary definition in [14].

Definition 39 (see [14]). A category $\mathbf{K}$ is called a cartesian category if it satisfies the following conditions.

(i) There is a terminal object.

(ii) Each pair of objects $D$ and $E$ of $\mathbf{K}$ has a product $D \times E$ with projections $p_{1}: D \times E \rightarrow D$ and $p_{2}: D \times E \rightarrow$ E.

(iii) Each pair of objects $D$ and $E$ of $\mathbf{K}$ has an exponentiation $E^{D}$, that is, an object $E^{D}$ and an arrow eval: $E^{D} \times D \rightarrow E$ with the property that for any $f$ : $X \times D \rightarrow E$, there is a unique arrow $\lambda_{f}: X \rightarrow E^{D}$ such that the composite

$$
X \times D \stackrel{\lambda_{f} \times D}{\longrightarrow} E^{D} \times D \stackrel{\text { eval }}{\longrightarrow} E
$$

is $f$.

Lemma 40. The cartesian product of two strongly continuous lattices is strongly continuous.

Proof. Let $D, E$ be strongly continuous lattices. Then $D, E$ are continuous lattices. By Proposition I-2.1 of [2], the product $D \times E$ of $D$ and $E$ is continuous. It is easy to verify that the projections $p_{1}: D \times E \rightarrow D, p_{2}: D \times E \rightarrow E$ are Scott continuous. Let $(a, b) \Leftarrow(c, d)$ in $D \times E$. Since $D, E$ are strongly continuous lattices, $\downarrow_{\ll} c \in R d(D), \downarrow_{\ll} d \in R d(E)$ and $(c, d)=\bigvee \downarrow_{\ll}(c, d)=\bigvee\left(\downarrow_{\ll} c \times \downarrow_{\ll} d\right)$. Since both $\downarrow_{\ll} c$ and $\downarrow_{\ll} d$ are semiprime ideals, it follows that $\downarrow_{\ll} c \times \downarrow_{\ll} d$ is the semiprime ideal of $D \times E$. Hence $(a, b) \ll(c, d)$. Thus $D \times E$ is strongly continuous by the Theorem 2.5 of [5]. By Corollary $28, p_{1}, p_{2}$ are semicontinuous. This completes our proof.

Theorem 41. The category SCONT is cartesian closed in which the exponential $E^{D}$ is $[D, E]$.

Proof. Note that the morphisms in SCONT are the Scott continuous mappings. Clearly the singleton set is a strongly continuous lattice and serves as a terminal object in SCONT. By Lemma 40, the cartesian product of two strongly continuous lattices is again a strongly continuous lattice.

By Theorem 38, $[D, E]$ is a strongly continuous lattice. Define eval: $E^{D} \times D \rightarrow E$ by

$$
\operatorname{eval}(h, a)=h(a) .
$$

Then eval is a Scott continuous mapping. Let $X$ be a strongly continuous lattice, let $f: X \times D \rightarrow E$ be a semicontinuous (or Scott continuous) mapping, and define $\lambda_{f}: X \rightarrow E^{D}$ by $\lambda_{f}(x)(a)=f(x, a)$. For any directed set $I \subseteq X$ and $a \in D$, $\lambda_{f}(\bigvee I)(a)=f(\bigvee I, a)=f(\bigvee I \times\{a\})=\bigvee\{f(x, a): x \in I\}$ because $I \times\{a\}$ is directed. Thus $\lambda_{f}(\bigvee I)(a)=\bigvee\left\{\lambda_{f}(x, a)\right.$ : $x \in I\}=\bigvee_{x \in I} \lambda_{f}(x)(a)$. So $\lambda_{f}(\bigvee I)=\bigvee \lambda_{f}(I)$. Thus $\lambda_{f}$ is Scott continuous. And eval $\circ\left(\lambda_{f} \times i d_{D}\right)(x, a)=$ eval $(f(x), a)=f(x)(a)=f(x, a)$. So eval $\circ\left(\lambda_{f} \times i d_{D}\right)=f$. Clearly $\lambda_{f}$ is the unique morphism satisfying the condition. By Definition 39, the category SCONT is cartesian closed. The proof is completed.

Remark 42. By [5], every distributive continuous lattice is strongly continuous. One can verify straightforwardly that for any two distributive continuous lattices $S$ and $T$, the lattice $[S, T]$ of $S$ cott continuous mappings from $S$ to $T$ is distributive and thus is a distributive continuous lattice. Hence one can prove, by a similar argument as for SCONT, that the fully subcategory DCONT of SCONT consisting of all distributive continuous lattices is cartesian closed.

\section{Acknowledgments}

This work has been supported by the National Natural Science Foundation of China (no. 11071061, no. 11201490, and no. 11226151), the National Basic Research Program of China (no. 2011CB311808), the Natural Science Foundation of Hunan Province under Grant no. 10JJ2001, and Science and Technology Plan Projects of Hunan Province no. 2012FJ3145.

\section{References}

[1] D. Scott, "Continuous Lattices," in Toposes, Algebraic Geometry and Logic (Conf., Dalhousie Univ., Halifax, N. S., 1971), vol. 274 of Lecture Notes in Math., pp. 97-136, Springer, Berlin, Germany, 1972.

[2] G. Gierz, K. H. Hofmann, K. Keimel, J. D. Lawson, M. Mislove, and D. S. Scott, Continuous Lattices and Domains, Springer, Berlin, Germany, 2003.

[3] A. Jung, "Cartesian closed categories of algebraic cpos," Theoretical Computer Science, vol. 70, no. 2, pp. 233-250, 1990.

[4] G. Gierz and J. D. Lawson, "Generalized continuous and hypercontinuous lattices," The Rocky Mountain Journal of Mathematics, vol. 11, no. 2, pp. 271-296, 1981.

[5] D. Zhao, "Semicontinuous lattices," Algebra Universalis, vol. 37, no. 4, pp. 458-476, 1997.

[6] R. C. Powers and T. Riedel, " $Z$-semicontinuous posets," Order, vol. 20, no. 4, pp. 365-371, 2003.

[7] Y. Liu and L. Xie, "On the category of semicontinuous lattices," Journal of Liaoning Normal University (Natural Science), vol. 20, no. 3, pp. 182-185, 1997 (Chinese).

[8] Y. Liu and L. Xie, "On the structure of semicontinuous lattices and cartesian closedness of categories of semicontinuous lattices," Journal of Liaoning Normal University (Natural Science), vol. 18, no. 4, pp. 265-268, 1995 (Chinese).

[9] X. H. Wu, Q. G. Li, and R. F. Xu, "Some properties of semicontinuous lattices," Fuzzy Systems and Mathematics, vol. 20, no. 4, pp. 42-46, 2006 (Chinese).

[10] X. H. Wu and Q. G. Li, "Characterizations and functions of semicontinuous lattices," Journal of Mathematical Research and Exposition, vol. 27, no. 3, pp. 654-658, 2007 (Chinese).

[11] Y. Rav, "Semiprime ideals in general lattices," Journal of Pure and Applied Algebra, vol. 56, no. 2, pp. 105-118, 1989.

[12] G. N. Raney, "Completely distributive complete lattices," Proceedings of the American Mathematical Society, vol. 3, pp. 677$680,1952$. 
[13] B. Zhao and D. Zhao, "Lim-inf convergence in partially ordered sets," Journal of Mathematical Analysis and Applications, vol. 309, no. 2, pp. 701-708, 2005.

[14] M. B. Smyth, "The largest Cartesian closed category of domains," Theoretical Computer Science, vol. 27, no. 1-2, pp. 109119, 1983. 


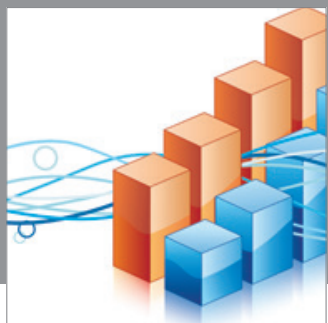

Advances in

Operations Research

mansans

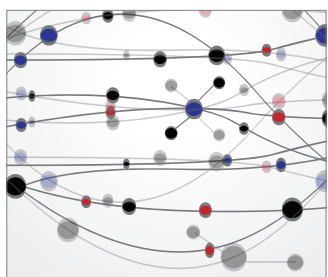

The Scientific World Journal
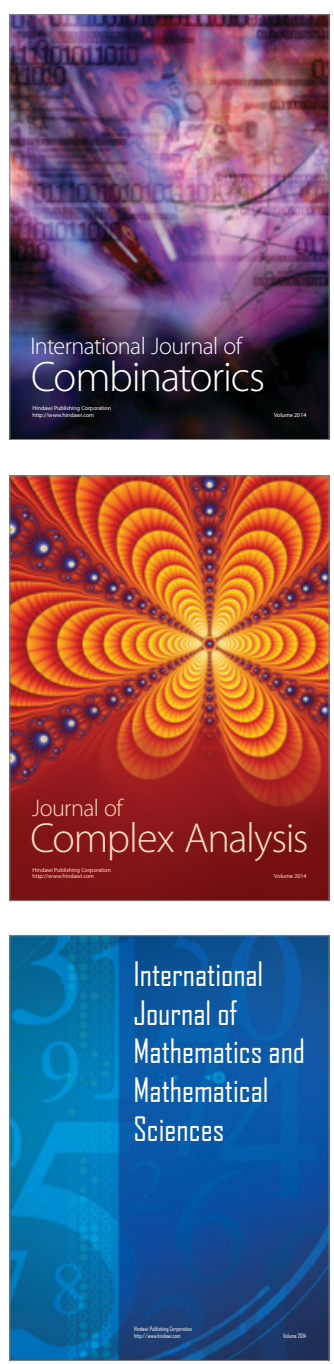
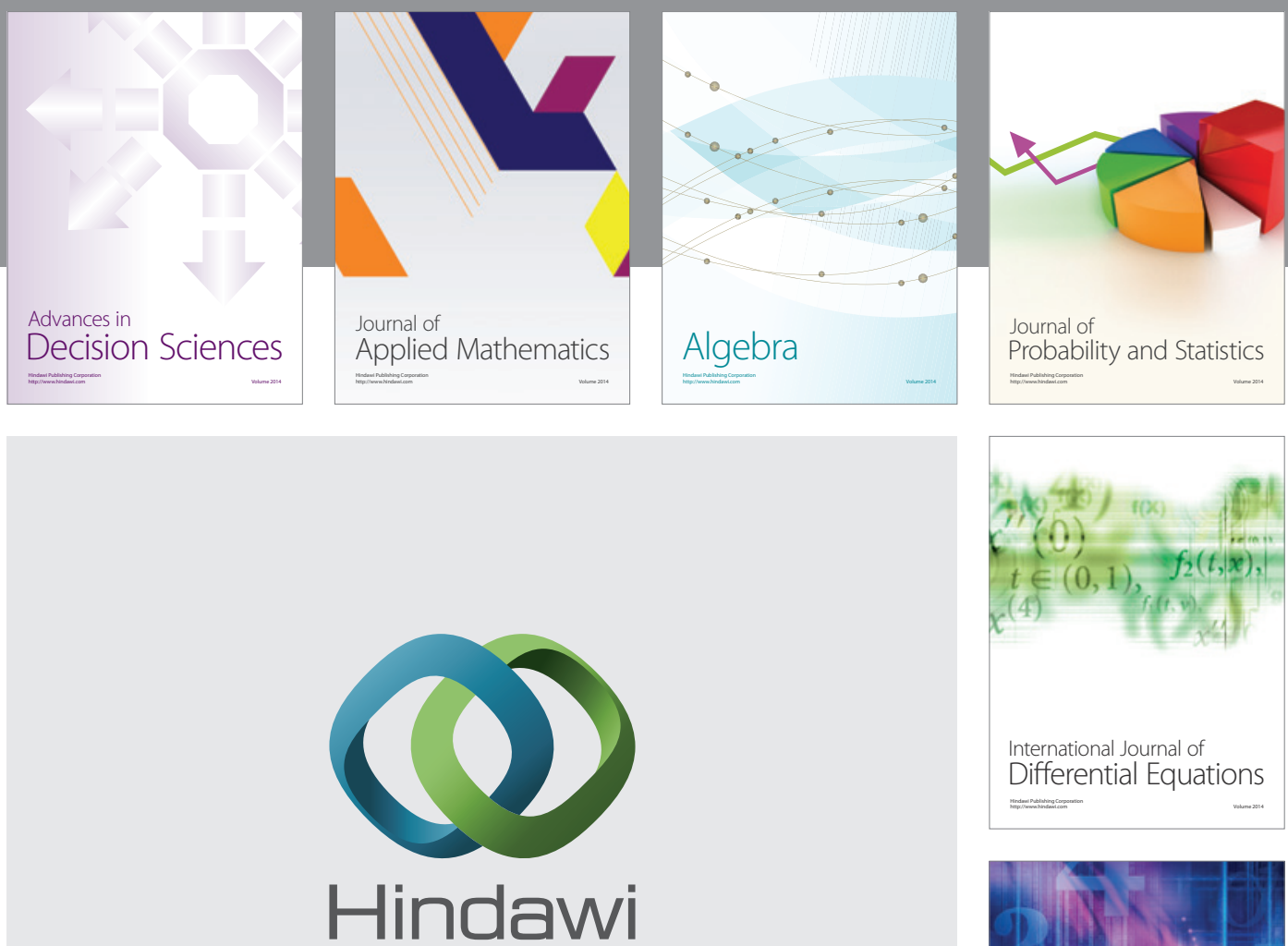

Submit your manuscripts at http://www.hindawi.com
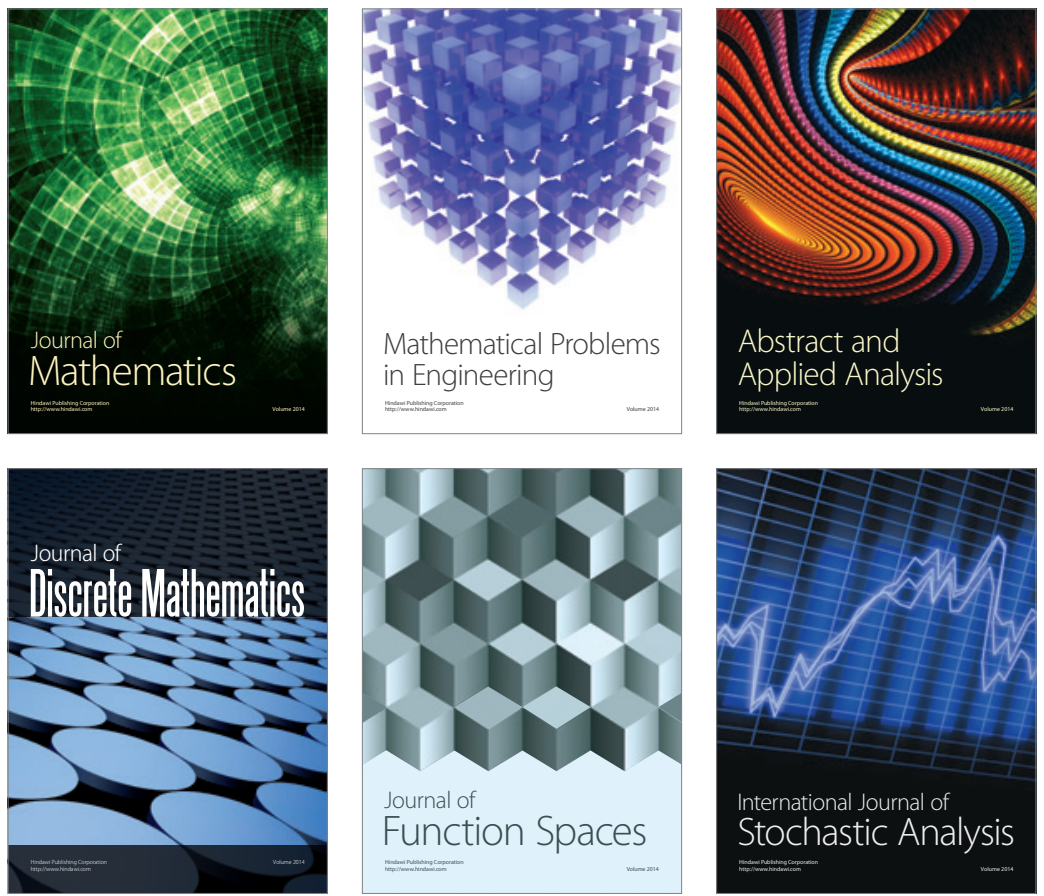

Journal of

Function Spaces

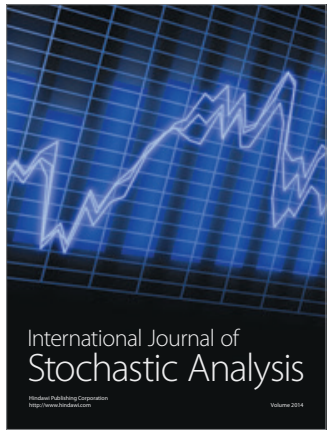

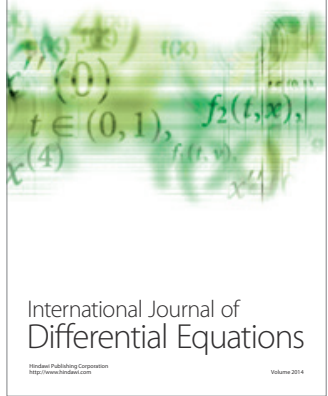
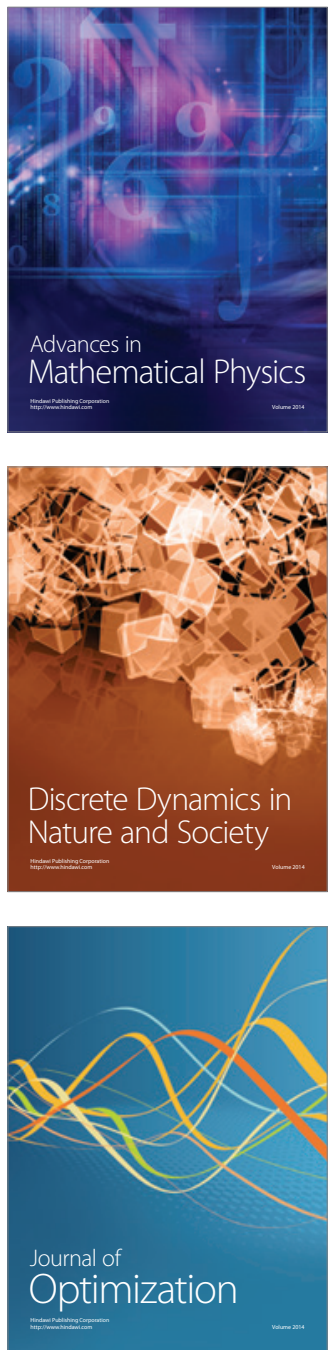